\title{
CIPPN: computational identification of protein pupylation sites by using neural network
}

\author{
Wenzheng Bao ${ }^{1, *}$, Zhu-Hong You ${ }^{2, *}$ and De-Shuang Huang ${ }^{1}$ \\ ${ }^{1}$ Institute of Machine Learning and Systems Biology, School of Electronics and Information Engineering, Tongji University, \\ Shanghai, China \\ ${ }^{2}$ Xinjiang Technical Institutes of Physics and Chemistry, Chinese Academy of Science, Urumqi 830011, China \\ *The first two authors should be regarded as joint First Authors \\ Correspondence to: De-Shuang Huang, email: dshuang@tongji.edu.cn \\ Keywords: disease; post translational modification; classification
}

Received: July 14, $2017 \quad$ Accepted: September 03, $2017 \quad$ Published: November 06, 2017

Copyright: Bao et al. This is an open-access article distributed under the terms of the Creative Commons Attribution License 3.0 (CC BY 3.0), which permits unrestricted use, distribution, and reproduction in any medium, provided the original author and source are credited.

\section{ABSTRACT}

Recently, experiments revealed the pupylation to be a signal for the selective regulation of proteins in several serious human diseases. As one of the most significant post translational modification in the field of biology and disease, pupylation has the ability to playing the key role in the regulation various diseases' biological processes. Meanwhile, effectively identification such type modification will be helpful for proteins to perform their biological functions and contribute to understanding the molecular mechanism, which is the foundation of drug design. The existing algorithms of identification such types of modified sites often have some defects, such as low accuracy and time-consuming. In this research, the pupylation sites' identification model, CIPPN, demonstrates better performance than other existing approaches in this field. The proposed predictor achieves Acc value of 89.12 and Mcc value of 0.7949 in 10-fold cross-validation tests in the Pupdb Database (http://cwtung.kmu.edu.tw/ pupdb). Significantly, such algorithm not only investigates the sequential, structural and evolutionary hallmarks around pupylation sites but also compares the differences of pupylation from the environmental, conservative and functional characterization of substrates. Therefore, the proposed feature description approach and algorithm results prove to be useful for further experimental investigation of such modification's identification.

\section{INTRODUCTION}

Post-translational modifications results in various human diseases such as cancers and autoimmune diseases, pernicious anemia, cardiovascular disease, cancer and neurodegenerative disorders. Protein plays the key roles in the field of biology and disease. Such modifications provide a fine-tuned control of protein functions in various types of cells in the field of disease research and drug design. For example, the well-known tumor suppressor p53 is subject to many post-translational modifications, which have ability to altering its localization, stability and other related functions, thus ultimately modulating its response to various forms of genotoxic stress [1-4]. Therefore, p53 drives both the activation and repression of a large number of promoters, which ultimately define its tumor sup-pressor abilities [5-10]. It could not be ignored that the above mentioned tumor suppressor is a critical transcription factor in the field of post translational modification [11].

When it comes to the post translational modification, it seems to be essential for regulating protein functions in all living cells and organisms [12-14]. It should be noted that ubiquitylation may seem to be one of the most common type of protein post-translational modification [15]. Such type plays significant roles in the regulation of 
DNA repair, transcription and other cellular processions. On the other hand, ubiquitylation is critical in the several types of Human diseases, such as lung cancer, breast cancer, Type 2 diabetes and other complex diseases which have been serious threats to human health [16-20].

Recently, pupylation, which is a common modification type in the protein post translational modification, has been treated as the first PTM in prokaryotes [21, 22]. Similar to ubiquitin, prokaryotic ubiquitin-like protein (Pup) seems to attach to specific lysine residues. As the initially found the PTM small protein modification in prokaryotes, prokaryotic ubiquitinlike protein (Pup) in Mycobacterium tuberculosis (Mtb) play an important role in the selection of proteins' degradation [23].

To better understand the biological mechanisms of pupylation, the basic target and fundamental task are the accurate and effective prediction of the pupylation sites. Another is worth mentioning, cellular pathways involved in determining the fate of essential proteins by PTM processions and events. Such pathways seem to be an increasingly important area of related study in the field. Among so many modifications, the better understanding of eukaryotic ubiquitylation by ubiquitin protein has shown to be especially essential and valuable [24-28]. With those capabilities and functionalities, such pathways play particular key roles in the cellular events [29-31].

Recently, several large-scale proteomics advanced technologies have been brought in identification pupylation sites [32-36]. Considering conventional experimental approaches' weakness is usually costly and luxury. Therefore, it is urgent to design and develop computational methods to identify the potential pupylation sites. Up to now, several predictors have been proposed and developed for such events. When it comes to the group-based prediction system 2.2 versions (GPS2.2) algorithm, Liu and their coworkers introduced the first predictor for the prediction of the pupylation sites in the field of bioinformatics [37]. Yan $\mathrm{Xu}$ and their team developed the iSulf-Cys algorithm to identify the S-sulfenylation Sites with the physicochemical properties of amino acid residues [38, 39]. Tung developed a predictor, which is named the iPUP server, utilizing the composition of $\mathrm{k}$-spaced amino acid pairs that are a special composition of amino acid and its abbreviation is CKSAAPs surrounding lysine-centered peptides with the SVM algorithm [40]. Chen and colleagues have designed a predictor on support vector machine named PupPred server, where the amino acid pair composition employed as the features so as to encode lysine-centered peptides [41]. Currently, Hasan and coworkers proposed a web server, which is named pbPUP, to predict pupylation modification sites with the method on profile-based CKSAAPs' feature [42, 43]. And such model is also employed the SVM model as the classifier.

\section{RESULTS}

By fusing three different and distinguish amino acid residues' component information approaches, a new ensemble classification framework named, has been established for predicting pupylation sites in protein sequences. To evaluate the performance of the proposed two features, several parameters, including Sn, Sp, Acc, MCC and AUC have been employed as the in this work. The following equations, which include from eq.(1) to eq.(4), have the ability to demonstrate the function of the above mentioned parameters. All experiments are performed on the personal computer with a $3.40 \mathrm{GHz}$ Intel(R) Core(TM) i7-3770M CPU and $16 \mathrm{G}$ bytes of memory.

$$
\begin{gathered}
S n=\frac{T P}{T P+F N} \\
S p=\frac{T N}{T N+F P} \\
A c c=\frac{T P+T N}{T P+F P+T N+F N} \\
M C C=\frac{T P * T N-F P * F N}{\sqrt{(T P+T N) *(T P+F N) *(T N+F P) *(T N+F N)}}
\end{gathered}
$$

Where, the TP means the true sample in positive set, the TN means the true sample in the negative one, the FP means the false sample in the positive and the FN means the false sample in the negaitve. Meanwhile the AUC means the area under the ROC curse, which have the ability to show the receiver operating characteristic in the field of classification issue.

\section{Performance of AAIndex PCA}

In our study, each type of features has contributed to the prediction model in different degrees. So, the employed feature types' comparison showed in the Table 1. From the table, it was easily to find that the features on the amino acid upstream/downstream residues composition information play less significant effect in the pupylation sites prediction. In other words, the adjacent amino acid residues' statistic features do not meet the needs on accurate and precise prediction pupylation sites. The second type of classification feature is the features derived from the AAIndex. These features contain the physical, chemical and biological properties of each kind amino acid residues. From the table, we can find that the candidate properties work well in this kind of post translational modification. However, the large amount of pupylation segments will cause the huge number of feature information. Such situation will also bring the unprecedented challenges in the field of computation, storing and transmission. The next type of feature is the 
Table 1: Prediction the database on Pupdb 10-fold with AAIndex PCA

\begin{tabular}{lccccc}
\hline Subset & Sn(\%) & Sp(\%) & Acc(\%) & \multicolumn{1}{c}{ Mcc } & 0.6491 \\
\hline 1 & 65.21 & 96.45 & 80.83 & 0.7049 & 0.8115 \\
2 & 73.42 & 95.36 & 84.39 & 0.6980 & 0.8231 \\
3 & 69.43 & 97.56 & 83.50 & 0.6398 & 0.7667 \\
4 & 64.43 & 96.23 & 80.33 & 0.7291 & 0.8091 \\
5 & 72.02 & 98.32 & 85.17 & 0.6656 & 0.8073 \\
6 & 65.32 & 97.67 & 81.50 & 0.6912 & 0.8137 \\
7 & 68.64 & 97.53 & 83.09 & 0.7117 & 0.8342 \\
9 & 69.43 & 98.64 & 84.04 & 0.6970 & 0.8451 \\
10 & 67.57 & 98.67 & 83.12 & 0.7806 & 0.6987 \\
\hline
\end{tabular}

The first column records sensitivity of these ten subsets of the Pupdb. The second column records the specialty of such subsets. And the 3th and 4th column record the accuracy and the Markovian correlation coefficient, AUC of these data, respectively.

AAIndex features' combination by PCA. Such type of features seem to have the similar performances with the second features' type. It was noted that the scale of these features is far smaller than the former one. Therefore, the AAIndex features' combination with PCA has the ability to replacement the AAIndex features' combination in some degree. Meanwhile, the PCA procession merely survives the main information of former combination. Some minor information of the AAIndex features' combination will be taken into account in the future research.

In the aspect of neural network, we selected the optimal number of hidden neurons by testing from 2 to 5 with the alternative layers ranging from 2 to 4 . The results of 10 -fold validation were shown in Figure 1. The other performances' measures are listed in Table 1.

\section{Comparison with other methods}

To demonstrate the performance of proposed model, we compare current prediction model with the other models. Meanwhile, we also carry the comparisons among $k$ nearest neighbors, support vector machine and Naïve Bayes classification algorithms in this work. The testing set was submitted to the GPS-PUP web server and the outputs were utilized to calculate the corresponding sensitivity, specificity and other performance indicators. It should be pointed out that we can guarantee that the testing data's protein segments are not included in the training dataset of GPS-PUP.

During this work, it is found that the ensemble model affected by the random initializations similar to other machine learning algorithms. And then, we have repeated the experiments for several times with different initializations to demonstrate the stability of the proposed ensemble algorithm.

On the other hand, it is also interesting to find from the Figure 2 that the number of hidden layers in the neural network of the proposed ensemble algorithm plays a critical role in its performance. Although this paper has tested a large range from 2 to 4 and selected 15 to construct our final classification model. Meanwhile, the selection of these parameters can also be applied independent. Hence, one of the important future research topics is to discover the size of hidden layers and hidden nodes with difference type data structures.

From the Table 1, we can find that the performances of feature AAIndex PCA can clear distinct the difference between the negative samples and the positive ones. It was pointed that the first proposed feature extracting method achieves the average Acc value of 83.41 in the PupDB data set, which can be treated as the benchmark data set in the field of identification pupylation sites. And the other performances on evaluating the method are Sn, Sp and Mcc, whose values are 69.55, 97.51 and 0.6987 , respectively. So, in this 10 -fold cross validation, the domain of Acc can range from $80.33 \%$ to 85.17 . Meanwhile the Sn's domain can range from $65.21 \%$ to $77.71 \%$. And the upper bound and the lower bound of $\mathrm{Sp}$ are $98.67 \%$ and $95.36 \%$, respectively. At the same time, it is easy to find out that the values of Sn are significantly higher than the Sp's values in each subset. And the ROC curves of each subset show in the Figure 2.

\section{Performance of AAIndex BLOSUM62 PCA}

From the Table 2, we can find that the performances of feature AAIndex BLOSUM62 PCA can clear distinct 


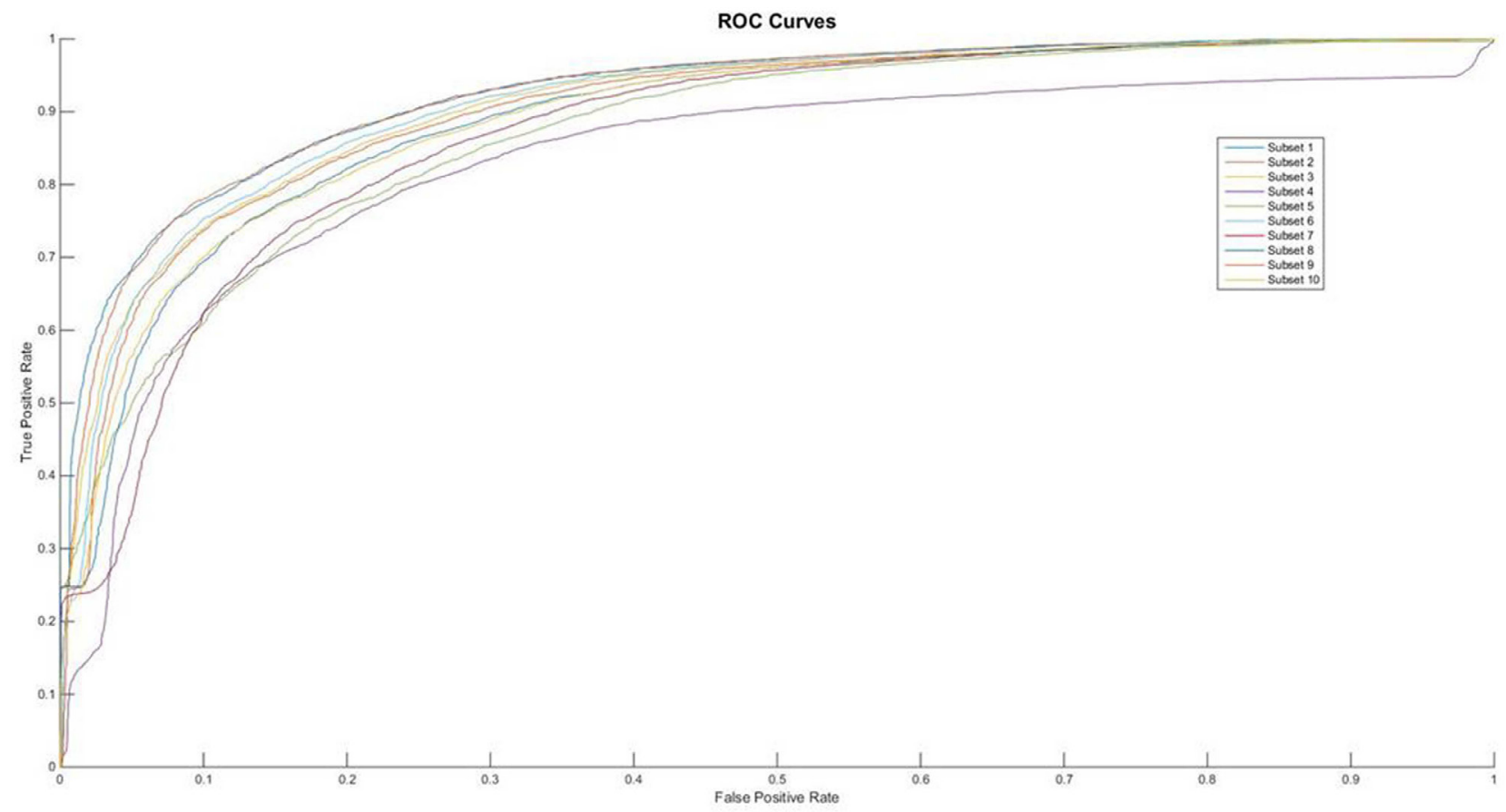

Figure 1: The ROC curves of feature of AAIndex PCA.

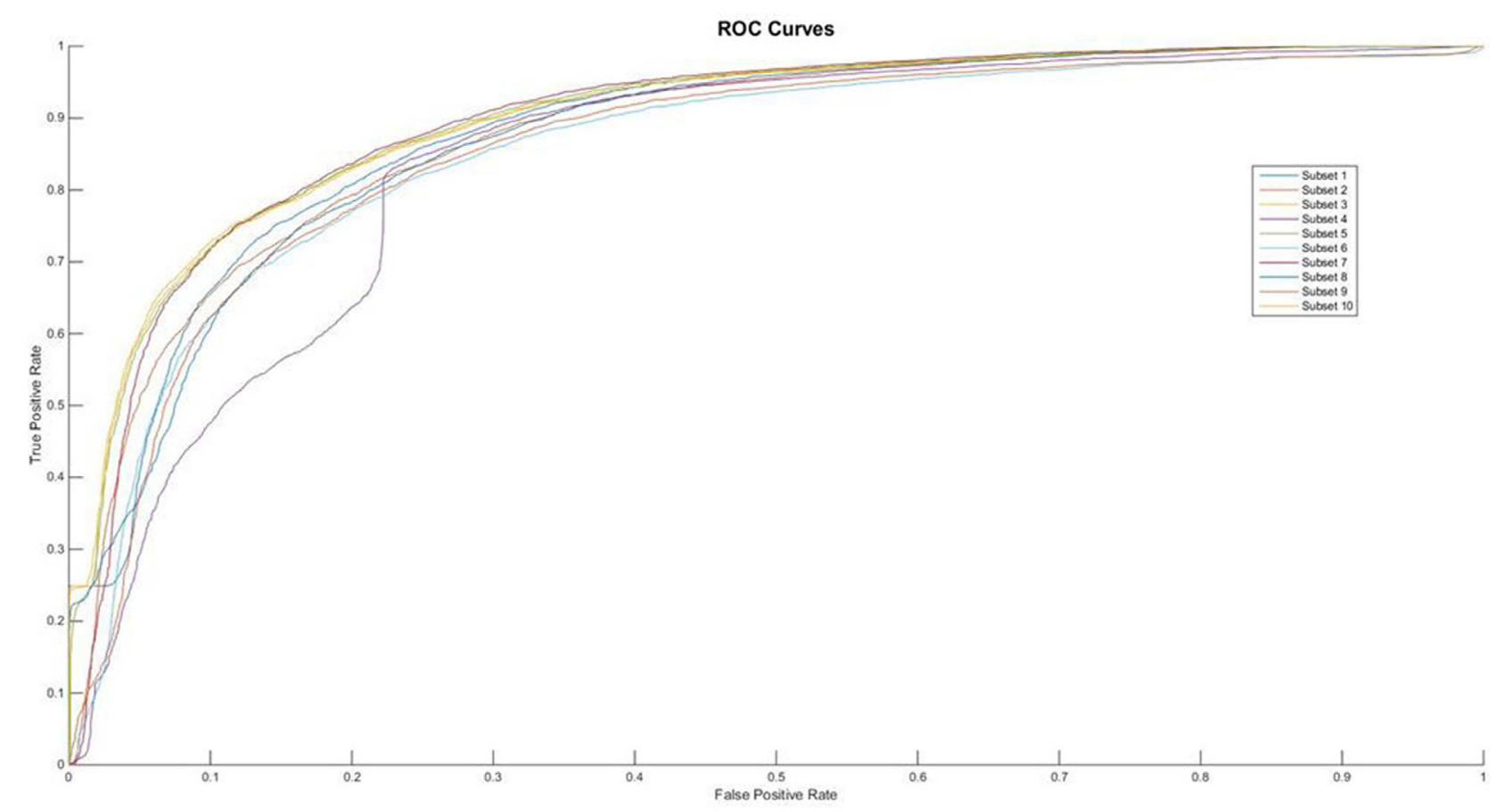

Figure 2: The ROC curves of feature of AAIndex BLOSUM62 PCA. 
Table 2: Prediction the database on Pupdb 10-fold with AAIndex BLOSUM62 PCA

\begin{tabular}{|c|c|c|c|c|c|}
\hline Subset & $\operatorname{Sn}(\%)$ & $\operatorname{Sp}(\%)$ & $\operatorname{Acc}(\%)$ & Mcc & AUC \\
\hline 1 & 99.81 & 80.48 & 90.15 & 0.8183 & 0.8127 \\
\hline 2 & 95.57 & 80.11 & 87.84 & 0.7660 & 0.8157 \\
\hline 3 & 99.27 & 76.79 & 88.03 & 0.7806 & 0.8287 \\
\hline 4 & 99.72 & 83.54 & 91.63 & 0.8437 & 0.7903 \\
\hline 5 & 99.34 & 81.59 & 90.46 & 0.8224 & 0.8107 \\
\hline 6 & 99.43 & 85.75 & 92.59 & 0.8599 & 0.8102 \\
\hline 7 & 99.52 & 77.53 & 88.52 & 0.7898 & 0.8167 \\
\hline 8 & 99.62 & 76.42 & 88.02 & 0.7817 & 0.8397 \\
\hline 9 & 99.75 & 80.47 & 90.11 & 0.8175 & 0.8576 \\
\hline 10 & 87.57 & 80.06 & 83.82 & 0.6782 & 0.8162 \\
\hline Average & 97.96 & 80.28 & 89.12 & 0.7949 & 0.8199 \\
\hline
\end{tabular}

The first column records sensitivity of these ten subsets of the Pupdb. The second column records the specialty of such subsets. And the 3th and 4th column record the accuracy and the Markovian correlation coefficient, AUC of these data, respectively.

Table 3: Prediction the Pupdb database comparison with other methods

\begin{tabular}{lccccc}
\hline Method & Sn(\%) & Sp(\%) & Acc(\%) & Mcc & AUC \\
\hline PUL-PUP & 82.24 & 91.57 & 88.92 & 0.7413 & 0.7238 \\
PSoL & 67.50 & 73.60 & 70.55 & 0.4118 & 0.6378 \\
SVM_balance & 76.71 & 63.65 & 69.88 & 0.4071 & 0.6571 \\
Nä̈ve Bayesian & 82.78 & 86.40 & 84.59 & 0.6923 & 0.7528 \\
DEC-SVM & 75.49 & 77.87 & 77.70 & 0.5338 & 0.7891 \\
SET-SVM & 93.77 & 77.87 & 79.05 & 0.7256 & 0.8013 \\
IMP-PUP & 94.58 & 78.12 & 79.34 & 0.7371 & 0.8031 \\
AAIndex & & & & & 0.8119 \\
PCA+Neural & 65.50 & 99.52 & 82.51 & 0.6914 & \\
Network & & & & & 0.8199 \\
AAIndex & & & & & \\
BLOSUM62 & 97.96 & 80.28 & & & \\
PCA+ Neural & & & & & \\
Network & & & & & \\
\hline
\end{tabular}

the differences between the negative samples and the positive ones. It was pointed that the first proposed feature extracting method achieves the average Acc value of 89.12 in the PupDB data set. And the other performances on evaluating the method are Sn, Sp and Mcc, whose values are $97.96,80.28$ and 0.7949 , respectively. So, in this 10 fold cross validation, the domain of Acc can range from $83.82 \%$ to $92.59 \%$. The range of Acc is much smaller than the AAIndex PCA method. Meanwhile the Sn's domain can range from $87.57 \%$ to $99.81 \%$. And the lower bound and the upper bound of $\mathrm{Sp}$ are $76.42 \%$ and $85.75 \%$, respectively. However, it is interesting to observe that the values of Sp are significantly higher than the Sn's values in each subset. And the ROC curves of each subset show in the Figure 2.

In order to evaluate the performance of those two methods, several pupylation identification methods and algorithm have been developed in the website resources. However, some of them had broken links, so they could hardly be tested in this model. In fact the predictors, which employed PUL-PUP, PSoL, SVM balance, Naïve Bayesian and other methods were included in the comparison tables. 
Table 4: The comparison with difference features

\begin{tabular}{lccccc}
\hline Features & Sn(\%) & Sp(\%) & Acc(\%) & Mcc & AUC \\
\hline Binary Encoding & 43.36 & 75.80 & 59.58 & 0.2026 & 0.6472 \\
AA Composition & 64.14 & 52.79 & 58.46 & 0.1704 & 0.6121 \\
AA Pair Composition & 62.46 & 62.48 & 62.47 & 0.2494 & 0.6917 \\
Grouping AA Composition & 41.78 & 76.04 & 58.91 & 0.1897 & 0.5919 \\
Physicochemical Properties & 55.53 & 63.93 & 59.73 & 0.1953 & 0.5976 \\
KNN Features & 64.94 & 55.85 & 60.39 & 0.2088 & 0.6477 \\
Secondary Tendency Structure & 59.96 & 57.40 & 58.68 & 0.1737 & 0.6211 \\
PSSM & 51.20 & 69.39 & 60.30 & 0.2094 & 0.6374 \\
Binary Coding & 64.04 & 78.60 & 71.63 & 0.4310 & 0.6271 \\
PSSM2 & 61.11 & 68.94 & 65.11 & 0.3014 & 0.7921 \\
AAIndex PCA & 65.50 & 99.17 & 82.32 & 0.6868 & 0.8119 \\
AAIndex BLOSUM62 PCA & 97.96 & 80.28 & 89.12 & 0.7949 & 0.8199 \\
\hline
\end{tabular}

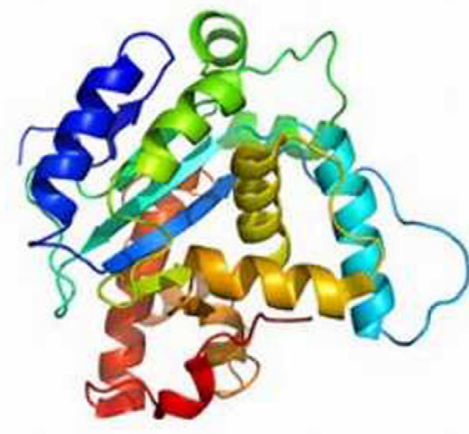

Protein Sequences

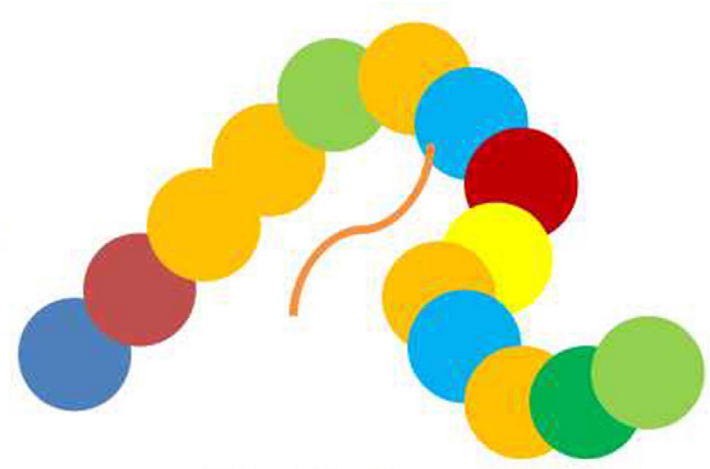

Protein Segments

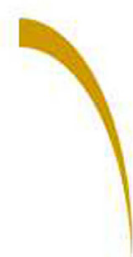

AAIndex PCA
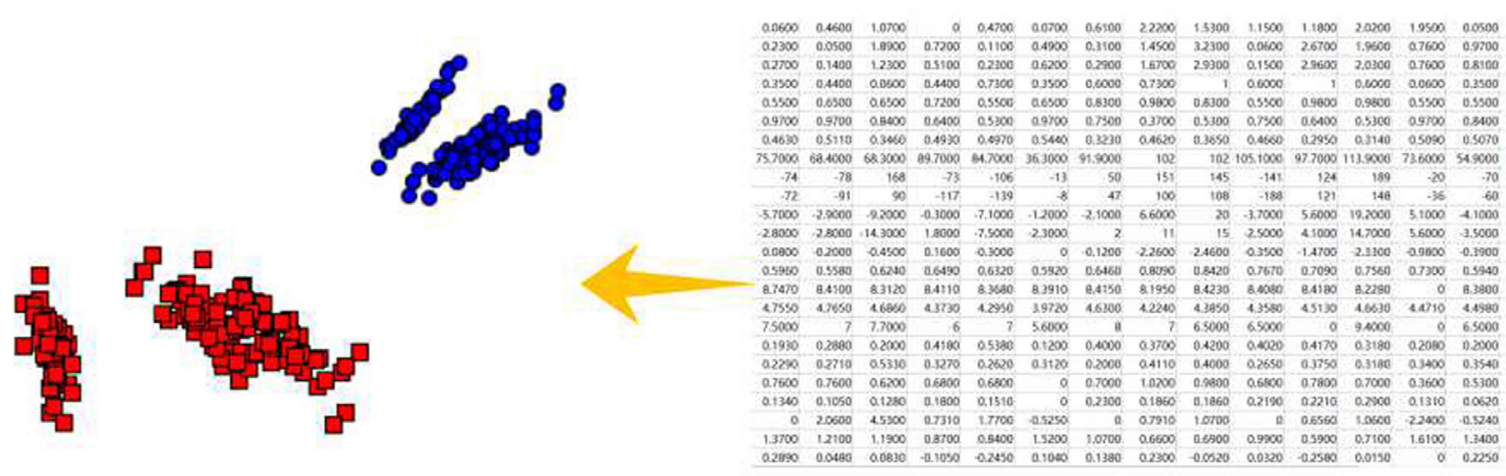

Principal Component Analysis

\section{Property}

Figure 3: The Steps of AAIndex PCA Features. The initial step is the predicted protein sequences in this work. The second step is the predicted amino acid segments from the protein sequences. The 3 th step is transform the amino acid segments to property matrix of the amino acid segments. The fourth step is the Principal Component Analysis (PCA) of the property matrix. 
Table 5: The selected properties from the AAIndex database

\begin{tabular}{lll}
\hline No. & AAIndex ID & Name of Properties \\
\hline 1 & CHOP780207 & Normalized frequency of C-terminal non helical region \\
2 & DAYM780201 & Relative mutability \\
3 & EISD860102 & Atom-based hydrophobic moment \\
4 & FAUJ880108 & Localized electrical effect \\
5 & FAUJ880111 & Positive charge \\
6 & FINA910103 & Helix termination parameter at position $\mathrm{j}-2, \mathrm{j}-1, \mathrm{j}$ \\
7 & JANJ780101 & Average accessible surface area \\
8 & KARP850103 & Flexibility parameter for two rigid neighbors \\
9 & KLEP840101 & Net charge \\
10 & KRIW710101 & Side chain interaction parameter \\
11 & KRIW790102 & Fraction of site occupied by water \\
12 & NAKH920103 & AA composition of EXT of single-spanning proteins \\
13 & QIAN880101 & Weights for alpha-helix at the window position of -6 \\
\hline
\end{tabular}
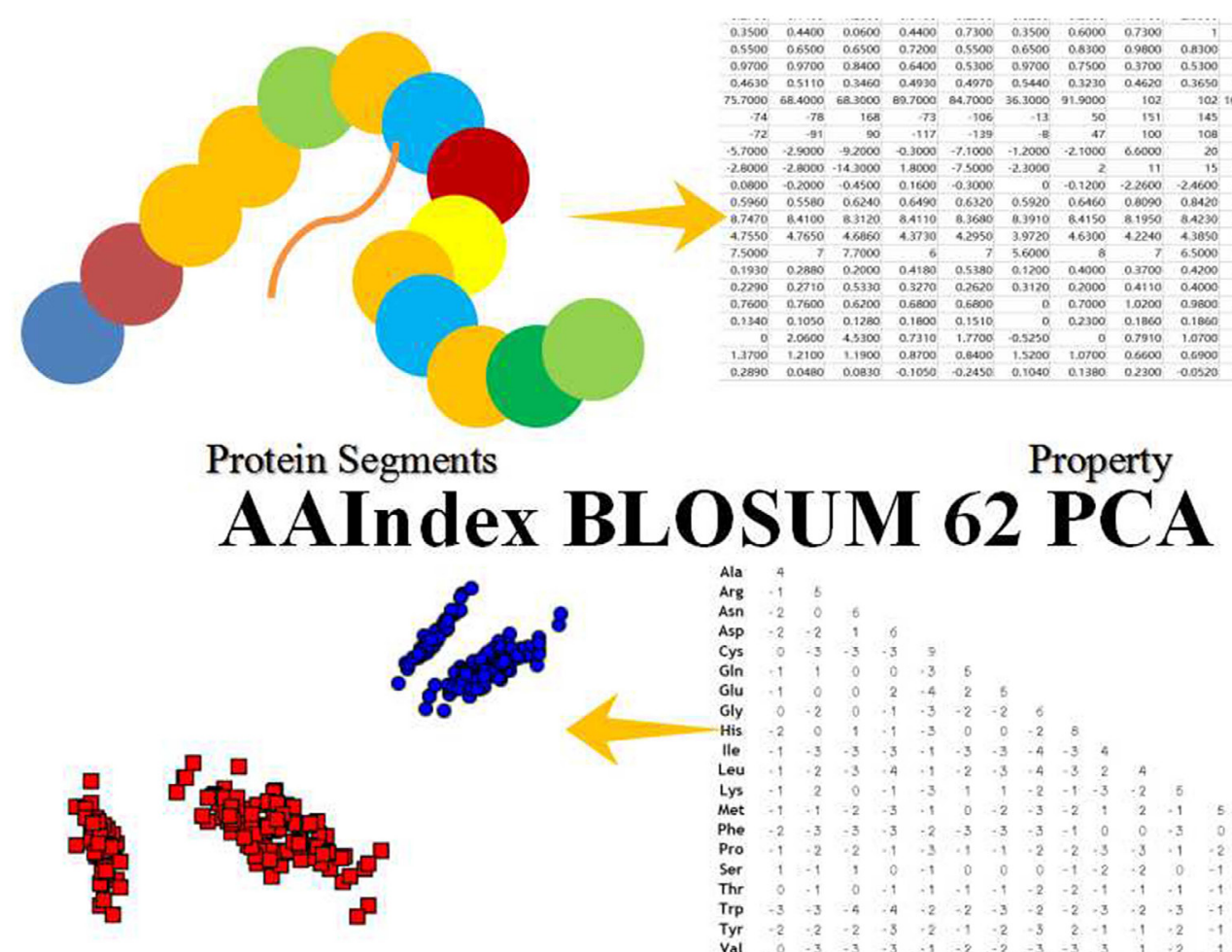

Principal Component Analysis

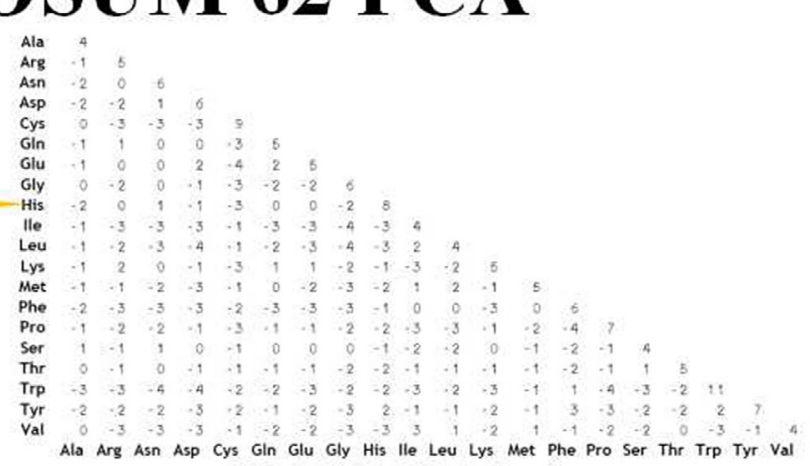

BLOSUM62 Matrix

Figure 4: The Steps of AAIndex BLOSUM62 PCA Features. The initial step is the protein segments of the predicted amino acid segments in this work. The 2nd step is transform the amino acid segments to property matrix of the amino acid segments. The first and second steps are same as the second and third steps of the steps of AAIndex PCA features. The 3th step is the BLOSUM 62 matrix, which is the interaction between the amino acid residues. The property matrix and the BLOSUM 62 matrix get the multiplication operation in this steps. And then, they get a novel interaction matrix. The fourth step is the Principal Component Analysis (PCA) with the novel interaction matrix. 
From the Table 3, we can find that the second proposed method can reach higher accuracy than the PUL_PUP method and the first method merely reach $82.51 \%$ in this performance. At the same time, we can also find that the methods such as the SET-SVM, IMP_PUP and the second method can get ideal values in the sensitivity and the methods such as the PUL-PUP, Naïve Bayesian and the first method can get appropriate value in the specialty.

In order to evaluate the performance of those two features, several pupylation identification features also have been developed in the literature resources. In this work, several features such as Binary Encoding, AA Composition, AA Pair Composition, Grouping AA Composition, Physicochemical Properties, KNN Features, Secondary Tendency Structure and Binary Coding have been compared. The comparison among these features show in the Table 4.

\section{DISCUSSIONS}

\section{Features}

Generally, the types of proteins' features can reach more than 10,000. Such huge of features, including statistical features such as amino acid compositions (AAC), dipeptide compositions (DC), biological features such as pseudo amino acid compositions (PseAAC), characteristic features such as hydrophilic, free energy of molecules and Van der Waals forces of amino acid residues and physical features such as relative molecular mass, molecular charge number and other relative features merely contain remarkably few key classification information in the prediction issue [68-70]. Nevertheless, the above mentioned features can hardly effectively and accurately have the ability to description the interaction between predicted modification lysine residue and upstream/downstream amino acid residues [71]. Therefore, a special type of features, utilized to classify and distinguish the pupylated lysine residues and the nonpupylated lysine residues, has been improved and polished in the proposed prediction method in this work.

Because of the potential sites, the features of amino acid residues should be taken into account. The most popular and well-known amino acids' feature index is the AAIndex, which is a website database of numerical indices representing various physical, chemical and biological properties of the amino acid residues, pairs of amino acid peptides, other forms of protein sequence information. All those relative information could be easily derived from published literatures [72-74]. So, several types of amino acids' features have been employed in this research. And the more detailed information on the selected amino acid features showed in Table 5.

In this work, we have selected several properties, which show in the Table 5, from the AAIndex database. Those selected properties have been constructed a matrix, whose size is $\mathrm{m}$ lines and $n$ columns. The $\mathrm{m}$ lines mean the $m$-length predicted protein segment and the n-columns mean the n-dimension selecting property in this research. However, the property matrix seems to be hardly treated as the feature in this classification model. Therefore, the PCA (Principal Components Analysis) has been employed as the feature processing. PCA is a mathematical algorithm that tries to reduce and decrease the dimensionality of the data matrix. The detailed steps show in Figure 3.

Given a predicted sample matrix with $\mathrm{m}$ amino acid residues and $\mathrm{n}$ properties, the matrix is first focused on the means of variables. This will make sure the data have the ability to centering on the origin of principal components, and the data could not be affected by the spatial relationships of the data nor the variances along other variables [74-76]. The principal components $Y$ is given by the linear combination of the variables $x_{1}, x_{2}, \ldots$, $x_{\mathrm{m}}$ and the formulate shows in the (5).

$$
Y=a_{1} x_{1}+a_{2} x_{2}+\cdots+a_{m} x_{m}
$$

The principal component is computed such that it accounts for the most possible variance of the selected properties. To prevent such state, weights are evaluated by the constraint that the sum of squares is equal to 1 . And the formulate shows in the (6).

$$
a_{1}^{2}+a_{2}^{2}+\cdots+a_{m}^{2}=1
$$

In this paper, we took advantage of the BLOSUM62 matrix, a popular substitution matrix used for sequence alignment of proteins. This explains some details in BLOSUM62 that may seem counter intuitive at first glance. For example, W/W combination score +11 and $\mathrm{L} / \mathrm{L}$ pair only score +4 . The scores could be evaluated by the following equation. Those scores consist of a $20 \times 20$ score matrix. In our work, the values of BLOSUM62 are treated as the weights between the potential predicted lysine sites and the adjacent amino acid residues. The second type of feature is the first type feature with the relation weight between the lysine and other kinds of amino acid residues in the predicted protein segments. In order to show the steps more clearly, the following Figure 4 will be described the steps.

\section{MATERIALS AND METHODS}

\section{Data}

The post translational modification resources show the detailed system flow of the online-construction. Considering the inaccessibility of database, it contents in several online PTM resources, 11 biological databases related to PTMs are integrated in dbPTM totally and several biological processions [44-47].

First of all, a series of keywords, which is related to the PTM-related terms, have been constructed by referring 
to the UniProtKB and SwissProt resources on the PTM list $[21,48,49]$. At the same time, the detailed information of those databases has been annotated by the RESID that is another international protein database in the field of proteomics [50-54].

Next, all fields could be searched by a series of keyword list of the constructed table list in the PubMed and other proteomics databases. According to not complete count, to 2016, about 850 review and original articles associated with $\mathrm{MS} / \mathrm{MS}$ proteomics and protein modifications are retrieved from those database. Therefore, those datasets of pupylated proteins and pupylation sites identified by large-scale proteomics experiments are extracted from various PTM databases [55]. Particularly, PupDB, which is a collection of pupylated proteins and pupylation sites, have been constructed by Tung and co-workers in 2012 [56, 57]. Such database includes 76, 51 and 55 pupylated proteins with known and reported pupylation sites in many datasets. Considering pupylation's occurrence on lysine residues, both positive and negative sample groups with silicon methods are represented as $2 m+1$ length residues' peptide segments with lysine in the center. The potential peptides with pupylated lysine in the center could be treated as positive samples. On the contrary, the other non-modified potential peptides seem to be the negative ones. At the same time, another step of the preprocessing seems to avoid overestimating prediction performances of proposed methods in this work. So the redundant peptides of identical sequences have been removed.

To solve the heterogeneity among those data collected from different databases from the website resource, such reported sites have been mapped from the UniProtKB protein. With the development of highthroughput of MS-based approaches in the field of posttranslational proteomics, this update, meanwhile, includes manually curated MS/MS-identified peptides associated with PTMs from research articles [58-63].

The source of pupylation protein sequences have been extracted by the UniProtKB/Swissprot database in this research [64]. To ensure the quality, the selected data, have been used in this research, was constructed by the UniProtKB/SwissProt at http://www.ebi.ac.uk/uniprot/.

The detailed procedures show as following the steps:

I. Vistiting the website at http://www.uniprot.org/, and then the button 'Advanced'.

II. Choosing the 'Modified residue' for 'Fields'.

III. Choosing the 'Any experimental assertion' for 'Evidence'.

IV. The proteins thus obtained were subject to a screening operation to remove those sequences, which have above $50 \%$ pairwise sequence identity to any other.

It was pointed that the aforementioned existing prediction servers were generally trained about the experimentally annotated pupylated proteins. However, those prediction servers' data resources have been collected from the PupDB database, which is a classical benchmark database [6]. It is noteworthy that only 268 annotated pupylated proteins with 311 known pupylation sites were included in the current version of PupDB database [65-67]. Considering such phenomenon, the scale of defined and submitted the modified protein sequences seem to be relatively small. Those prediction models and relative researcher could hardly reflect the real distribution of modification sites commendably. Consequently, the prediction accuracy of existing computational methods could hardly be unsatisfactory. Really, there are 268 annotated pupylated protein sequences.

In this study, the proposed method, which aims to improve the prediction of pupylation sites, by using an alternative structure neural network and employed two types of protein information as the classification features. Specifically, the alternation structure neural network classification model is trained on those training proteins segments taking advantage of the selected features. And the initial ensemble model is utilized to classification the testing pupylated proteins segments. Then, the final ensemble classification, which is used to construct the proposed algorithm, results at the end of classification. As illustrated by our experimental results, the performance of the predictor has been improved effectively by the selected data set. The results indicated that the proposed algorithm outperforms three other existing predictors significantly.

\section{CONCLUSIONS}

Much knowledge about protein sequences with pupylation has been accumulated to date. There are still numerous unanswered issues and questions regarding specific aspects of the classification issue in the field of machine learning. Nowadays non-consensus sequences that make up their mind which specific lysine would become pupylation could be identified when nonhomologous proteins seem to be considered. It is hard to regard that all segments carry similar structures before they bind to the component of the pupylation modification.

Systematic analysis of the pupylated sites along with information on the exact sites is utilized by identifying the modified sites from the protein sequences. Here, it can be easily find that not only the sequence markers but also structural markers about pupylated sites. First of all, the analysis of sequence features demonstrates that the adjacent amino acid residues in the potential segments could be close to modified lysines residues in spatial structure. Secondly, pupylation protein segments have high propensity flexibility in the field of protein structure. Finally, the conservative in pupylation segments seem to be high.

On the other hand, another significant result of this research is design of the pupylation sites prediction model with different types of features. Every selected type of features is contributed to the prediction model 
more or less. Here, it was pointed that unbalanced datasets, which the negative samples can reach 5 times than the positive ones, present a hottest topic in the field of machine learning classification. In our work, the unbalanced datasets will try to avoid the negative impacts with the preprocess steps, which the positive samples replicate themselves until the size of positive samples can generally reach the scale of the negative ones in both training and testing set. Nevertheless, the preprocess method will increase the burden of classification model. The model's training time will be greatly extended. Considering the burden and the training time, an improved preprocess step has been introduced to deal with the unbalance classification model. Such improved step merely replicate the positive samples in the testing set. With such step, the unbalanced classification issues can be solved basically and the burden of classification model will not increase. For future research, other methods, such as semi-supervised learning, will be explored and developed to deal with the unlabeled post translational modification sites in the predicted protein segments.

To summarize, the design of ensemble classification model represents an attempt to predict candidate pupylated segments based on the multi-type feature. Because the size of experimentally identified modification sites will be rocketing in the future and such sites will be enriched the training set, the current accuracy of the ensemble is helpful to identify the new sites. With the established link between the feature description and the classification system, such predictions, especially when confirmed by experiments, would be helpful to identify the degradation possibilities of individual proteins more precisely, and may ultimately lead to design of drugs and treatment of diseases.

In this work, we have developed a novel pupylation sites prediction ensemble algorithm. To our knowledge, it is the first time such ensemble flexible neural tree model has been applied to predict the potential pupylation sites. Experimental results demonstrate that such method outperformed the existing pupylation sites prediction. At the same time, the majority modification type likely pupylation sites could be predicted in non-annotated lysine sites by utilizing the proposed ensemble model. Meanwhile, it could be believed that such method can be utilized to prediction the other types of modified sites in the potential protein segments. Therefore, we will design and develop the web server for such algorithm in future research.

\section{Author contributions}

Wen-Zheng Bao wrote the article and performed most of the experiments and data collection; De-Shuang Huang participated in discussion and article writing; ZhuHong You provided technical assistance.

\section{ACKNOWLEDGMENTS}

This work was supported by the grants of the National Science Foundation of China and China Postdoctoral Science Foundation.

\section{CONFLICTS OF INTEREST}

There is no conflicts of interest that I should disclose, having read the above statement.

\section{FUNDING}

This work was supported by the grants of the National Science Foundation of China, Nos. 61732012, 61520106006, 31571364, U1611265, 61532008, $61672382,61772370,61402334,61472282$, and 61472173 and China Postdoctoral Science Foundation [Grant No. 2015M580352, 2017M611619, and 2016M601646]. De-Shuang Huang is the corresponding author of this paper.

\section{REFERENCES}

1. Mann M, Jensen ON. Proteomic analysis of posttranslational modifications. Nat Biotechnol. 2003; 21:255-61.

2. Hornbeck PV, Kornhauser JM, Tkachev S, Zhang B, Skrzypek E, Murray B, Latham V, Sullivan M. PhosphoSitePlus: a comprehensive resource for investigating the structure and function of experimentally determined post-translational modifications in man and mouse. Nucleic Acids Res. 2012; 40:D261-70.

3. Westermann S, Weber K. Post-translational modifications regulate microtubule function. Nat Rev Mol Cell Biol. 2003; 4:938-47.

4. Witze ES, Old WM, Resing KA, Ahn NG. Mapping protein post-translational modifications with mass spectrometry. Nat Methods. 2007; 4:798-806.

5. Walsh G, Jefferis R. Post-translational modifications in the context of therapeutic proteins. Nat Biotechnol. 2006; 24:1241-52.

6. Perkins ND. Post-translational modifications regulating the activity and function of the nuclear factor kappa B pathway. Oncogene. 2006; 25:6717-30.

7. Adamson P, Marshall CJ, Hall A, Tilbrook PA. Posttranslational modifications of $\mathrm{p} 21$ rho proteins. J Biol Chem. 1992; 267:20033-38.

8. Wells L, Vosseller K, Cole RN, Cronshaw JM, Matunis MJ, Hart GW. Mapping sites of O-GlcNAc modification using affinity tags for serine and threonine post-translational modifications. Mol Cell Proteomics. 2002; 1:791-804.

9. Hori Y, Kikuchi A, Isomura M, Katayama M, Miura Y, Fujioka H, Kaibuchi K, Takai Y. Post-translational 
modifications of the C-terminal region of the rho protein are important for its interaction with membranes and the stimulatory and inhibitory GDP/GTP exchange proteins. Oncogene. 1991; 6:515-22.

10. Janke C, Kneussel M. Tubulin post-translational modifications: encoding functions on the neuronal microtubule cytoskeleton. Trends Neurosci. 2010; 33:362-72.

11. Konstantinopoulos PA, Karamouzis MV, Papavassiliou AG. Post-translational modifications and regulation of the RAS superfamily of GTPases as anticancer targets. Nat Rev Drug Discov. 2007; 6:541-55.

12. Sims RJ 3rd, Reinberg D. Is there a code embedded in proteins that is based on post-translational modifications? Nat Rev Mol Cell Biol. 2008; 9:815-20.

13. Bode AM, Dong Z. Post-translational modification of $\mathrm{p} 53$ in tumorigenesis. Nat Rev Cancer. 2004; 4:793-805.

14. Deribe YL, Pawson T, Dikic I. Post-translational modifications in signal integration. Nat Struct Mol Biol. 2010; 17:666-72.

15. Garcia BA, Hake SB, Diaz RL, Kauer M, Morris SA, Recht J, Shabanowitz J, Mishra N, Strahl BD, Allis CD, Hunt DF. Organismal differences in post-translational modifications in histones H3 and H4. J Biol Chem. 2007; 282:7641-55.

16. Olsen JV, Mann M. Status of large-scale analysis of posttranslational modifications by mass spectrometry. Mol Cell Proteomics. 2013; 12:3444-52.

17. Faus H, Haendler B. Post-translational modifications of steroid receptors. Biomed Pharmacother. 2006; 60:520-28.

18. Iyer LM, Burroughs AM, Aravind L. Unraveling the biochemistry and provenance of pupylation: a prokaryotic analog of ubiquitination. Biol Direct. 2008; 3:45.

19. Poulsen C, Akhter Y, Jeon AH, Schmitt-Ulms G, Meyer HE, Stefanski A, Stühler K, Wilmanns M, Song YH. Proteomewide identification of mycobacterial pupylation targets. Mol Syst Biol. 2010; 6:386.

20. Imkamp F, Rosenberger T, Striebel F, Keller PM, Amstutz B, Sander P, Weber-Ban E. Deletion of dop in Mycobacterium smegmatis abolishes pupylation of protein substrates in vivo. Mol Microbiol. 2010; 75:744-54.

21. Liu Z, Ma Q, Cao J, Gao X, Ren J, Xue Y. GPS-PUP: computational prediction of pupylation sites in prokaryotic proteins. Mol Biosyst. 2011; 7:2737-40.

22. Burns KE, Darwin KH. Pupylation versus ubiquitylation: tagging for proteasome-dependent degradation. Cell Microbiol. 2010; 12:424-31.

23. Delley CL, Striebel F, Heydenreich FM, Özcelik D, WeberBan E. Activity of the mycobacterial proteasomal ATPase $\mathrm{Mpa}$ is reversibly regulated by pupylation. J Biol Chem. 2012; 287:7907-14.

24. Huang DS, Zhang L, Han K, Deng S, Yang K, Zhang H. Prediction of protein-protein interactions based on proteinprotein correlation using least squares regression. Curr Protein Pept Sci. 2014; 15:553-60.
25. Wang B, Huang DS, Jiang C. A new strategy for protein interface identification using manifold learning method. IEEE Trans Nanobioscience. 2014; 13:118-23.

26. Huang DS, Yu HJ. Normalized feature vectors: a novel alignment-free sequence comparison method based on the numbers of adjacent amino acids. IEEE/ACM Trans Comput Biol Bioinformatics. 2013; 10:457-67.

27. Lei YK, You ZH, Ji Z, Zhu L, Huang DS. Assessing and predicting protein interactions by combining manifold embedding with multiple information integration. BMC Bioinformatics. 2012 (Supp1 7); 13:S3.

28. Yu H, Huang D. Novel 20-D descriptors of protein sequences and it's applications in similarity analysis. Chem Phys Lett. 2012; 531:261-66.

29. You ZH, Lei YK, Gui J, Huang DS, Zhou X. Using manifold embedding for assessing and predicting protein interactions from high-throughput experimental data. Bioinformatics. 2010; 26:2744-51.

30. You ZH, Yin Z, Han K, Huang DS, Zhou X. A semisupervised learning approach to predict synthetic genetic interactions by combining functional and topological properties of functional gene network. BMC Bioinformatics. 2010; 11:343-343.

31. Bao W, Chen Y, Wang D. Prediction of protein structure classes with flexible neural tree. Biomed Mater Eng. 2014; 24:3797-806.

32. Küberl A, Fränzel B, Eggeling L, Polen T, Wolters DA, Bott M. Pupylated proteins in Corynebacterium glutamicum revealed by MudPIT analysis. Proteomics. 2014; 14:1531-42.

33. Tung CW. Prediction of pupylation sites using the composition of k-spaced amino acid pairs. J Theor Biol. 2013; 336:11-17.

34. Zhao X, Dai J, Ning Q, Ma Z, Yin M, Sun P. Positionspecific analysis and prediction of protein pupylation sites based on multiple features. Biomed Res Int. 2013; 2013:109549.

35. DeMartino GN. PUPylation: something old, something new, something borrowed, something Glu. Trends Biochem Sci. 2009; 34:155-58.

36. Deng SP, Zhu L, Huang DS. Mining the bladder cancerassociated genes by an integrated strategy for the construction and analysis of differential co-expression networks. BMC Genomics. 2015 (Suppl 3); 16:S4.

37. K. E. Burns, K. H. Darwin, "Pupylation: proteasomal targeting by a protein modifier in bacteria," Ubiquitin Family Modifiers and the Proteasome: Reviews and Protocols, 2012; pp. 151-160. https://doi. org/10.1007/978-1-61779-474-2_10.

38. $\mathrm{Xu} \mathrm{Y,} \mathrm{Ding} \mathrm{J,} \mathrm{Wu} \mathrm{LY.} \mathrm{iSulf-Cys:} \mathrm{Prediction} \mathrm{of}$ S-sulfenylation Sites in Proteins with Physicochemical Properties of Amino Acids. PLoS One. 2016; 11:e154237.

39. Poulsen C, Akhter Y, Jeon AH, Schmitt-Ulms G, Meyer HE, Stefanski A, Stühler K, Wilmanns M, Song YH. 
Proteome-wide identification of mycobacterial pupylation targets. Mol Syst Biol. 2010; 6:386-386.

40. Tung CW. Prediction of pupylation sites using the composition of k-spaced amino acid pairs. J Theor Biol. 2013; 336:11-17.

41. Chen X, Qiu JD, Shi SP, Suo SB, Liang RP. Systematic analysis and prediction of pupylation sites in prokaryotic proteins. PLoS One. 2013; 8:e74002.

42. Hasan MM, Zhou Y, Lu X, Li J, Song J, Zhang Z. Computational Identification of Protein Pupylation Sites by Using Profile-Based Composition of k-Spaced Amino Acid Pairs. PLoS One. 2015; 10:e0129635.

43. Chen YZ, Tang YR, Sheng ZY, Zhang Z. Prediction of mucin-type $\mathrm{O}$-glycosylation sites in mammalian proteins using the composition of $\mathrm{k}$-spaced amino acid pairs. BMC Bioinformatics. 2008; 9:101-101.

44. Zhang TL, Ding YS, Chou KC. Prediction protein structural classes with pseudo-amino acid composition: approximate entropy and hydrophobicity pattern. J Theor Biol. 2008; 250:186-93.

45. Yu HJ, Huang DS. Graphical representation for DNA sequences via joint diagonalization of matrix pencil. IEEE J Biomed Health Inform. 2013; 17:503-11.

46. Berezovsky IN, Kilosanidze GT, Tumanyan VG, Kisselev LL. Amino acid composition of protein termini are biased in different manners. Protein Eng. 1999; 12:23-30.

47. Andreeva A, Howorth D, Chandonia JM, Brenner SE, Hubbard TJ, Chothia C, Murzin AG. Data growth and its impact on the SCOP database: new developments. Nucleic Acids Res. 2008; 36:D419-25.

48. Tung CW, Ho SY. Computational identification of ubiquitylation sites from protein sequences. BMC Bioinformatics. 2008; 9:310.

49. Chernorudskiy AL, Garcia A, Eremin EV, Shorina AS, Kondratieva EV, Gainullin MR. UbiProt: a database of ubiquitylated proteins. BMC Bioinformatics. 2007; 8:126.

50. Kawashima S, Kanehisa M. AAindex: amino acid index database. Nucleic Acids Res. 2000; 28:374-374.

51. Chen X, Qiu JD, Shi SP, Suo SB, Liang RP. Systematic analysis and prediction of pupylation sites in prokaryotic proteins. PLoS One. 2013; 8:e74002.

52. Radivojac P, Vacic V, Haynes C, Cocklin RR, Mohan A, Heyen JW, Goebl MG, Iakoucheva LM. Identification, analysis, and prediction of protein ubiquitination sites. Proteins. 2010; 78:365-80.

53. Huang DS, Zhang L, Han K, Deng S, Yang K, Zhang H. Prediction of protein-protein interactions based on proteinprotein correlation using least squares regression. Curr Protein Pept Sci. 2014; 15:553-60.

54. Huang DS, Yu HJ. Normalized feature vectors: a novel alignment-free sequence comparison method based on the numbers of adjacent amino acids. IEEE/ACM Trans Comput Biol Bioinform. 2013; 10:457-67.
55. Ding CH, Dubchak I. Multi-class protein fold recognition using support vector machines and neural networks. Bioinformatics. 2001; 17:349-58.

56. Chen K, Kurgan LA, Ruan J. Prediction of protein structural class using novel evolutionary collocation-based sequence representation. J Comput Chem. 2008; 29:1596-604.

57. Jones DT. Protein secondary structure prediction based on position-specific scoring matrices. J Mol Biol. 1999; 292:195-202.

58. Altschul SF, Madden TL, Schäffer AA, Zhang J, Zhang Z, Miller W, Lipman DJ. Gapped BLAST and PSI-BLAST: a new generation of protein database search programs. Nucleic Acids Res. 1997; 25:3389-402.

59. Kurgan LA, Zhang T, Zhang H, Shen S, Ruan J. Secondary structure-based assignment of the protein structural classes. Amino Acids. 2008; 35:551-64.

60. Kurgan L, Cios K, Chen K. SCPRED: accurate prediction of protein structural class for sequences of twilight-zone similarity with predicting sequences. BMC Bioinformatics. 2008; 9:226.

61. Liu T, Jia C. A high-accuracy protein structural class prediction algorithm using predicted secondary structural information. J Theor Biol. 2010; 267:272-75.

62. Huang DS, Zheng CH. Independent component analysis-based penalized discriminant method for tumor classification using gene expression data. Bioinformatics. 2006; 22:1855-62.

63. Huang DS, Jiang W. A general CPL-AdS methodology for fixing dynamic parameters in dual environments. IEEE Trans Syst Man Cybern B Cybern. 2012; 42:1489-500.

64. Lempel A, Ziv J. On the complexity of finite sequences. IEEE Trans Inf Theory. 1976; 22:75-81.

65. Ding S, Zhang S, Li Y, Wang T. A novel protein structural classes prediction method based on predicted secondary structure. Biochimie. 2012; 94:1166-71.

66. Li ZR, Lin HH, Han LY, Jiang L, Chen X, Chen YZ. PROFEAT: a web server for computing structural and physicochemical features of proteins and peptides from amino acid sequence. Nucleic Acids Res. 2006; 34:W32-7.

67. Rao HB, Zhu F, Yang GB, Li ZR, Chen YZ. Update of PROFEAT: a web server for computing structural and physicochemical features of proteins and peptides from amino acid sequence. Nucleic Acids Res. 2011 (suppl_2); 39:W385-90.

68. Chatterjee P, Basu S, Nasipuri M. Improving prediction of protein secondary structure using physicochemical properties of amino acids. Proceedings of the International Symposium on Biocomputing 2010; 10. https://doi. org/10.1145/1722024.1722036

69. Yang B, Chen Y, Jiang M. Reverse engineering of gene regulatory networks using flexible neural tree models. Neurocomputing. 2013; 99:458-66. 
70. Chen Y, Yang B, Dong J. Evolving flexible neural networks using ant programming and PSO algorithm. Advances in Neural Networks-ISNN. 2004; 2004:211-16.

71. Ding YS, Zhang TL, Chou KC. Prediction of protein structure classes with pseudo amino acid composition and fuzzy support vector machine network. Protein Pept Lett. 2007; 14:811-15.

72. Kawashima S, Pokarowski P, Pokarowska M, Kolinski A, Katayama T, Kanehisa M. AAindex: amino acid index database, progress report 2008. Nucleic Acids Res. 2008; 36:D202-05.

73. Kawashima S, Ogata H, Kanehisa M. AAindex: amino acid index database. Nucleic Acids Res. 1999; 27:368-69.
74. Zhao X, Ning Q, Chai H, Ma Z. Accurate in silico identification of protein succinylation sites using an iterative semi-supervised learning technique. J Theor Biol. 2015; 374:60-65.

75. Chou KC, Shen HB. Recent progress in protein subcellular location prediction. Anal Biochem. 2007; 370:1-16.

76. Shen HB, Chou KC. Predicting protein subnuclear location with optimized evidence-theoretic K-nearest classifier and pseudo amino acid composition. Biochem Biophys Res Commun. 2005; 337:752-56. 